\title{
Angiotensin converting enzyme in patients with sleep apnoea syndrome: plasma activity and gene polymorphisms
}

\section{To the Editor:}

In a recent issue of the European Respiratory Journal, BARCELO et al. [1] reported results on the plasma angiotensin converting enzyme (ACE) activity and the distribution of an insertion (I)/deletion (D) polymorphism of the ACE gene both in obstructive sleep apnoea syndrome (OSAS) patients and in healthy controls. They found that ACE activity was higher in OSAS patients $(53.9 \pm 2.5$ international units $\left.(\mathrm{IU}) \cdot \mathrm{L}^{-1}\right)$ than in healthy controls $\left(42.4 \pm 3.1 \mathrm{IU} \cdot \mathrm{L}^{-1}\right.$, $\mathrm{p}<0.01)$. However, the frequency distribution of the DD, II and ID genotypes in OSAS patients $(30 \%$, $16 \%, 54 \%$, respectively) was not significantly different from that seen in healthy subjects $(31 \%, 28 \%, 41 \%$, respectively, $\mathrm{p}=0.356$ ). They speculated that the increased ACE plasma activity contributed to the pathogenesis of the cardiovascular disease in these patients. The authors also described that "this is the first study investigating ACE in patients with OSAS". This is true, but the current study was not the first study examining the association of ACE gene polymorphism with OSAS.

XIAO et al. [2] firstly reported that the distribution of the DD, ID, and II ACE genotypes was $16 \%, 52 \%$, and $32 \%$ in the control subjects and $0 \%, 56 \%$, and $44 \%$ in OSAS patients, respectively. Although they did not measure ACE activity in the patients, they suggested that the II genotype and I allele might be a risk factor for OSAS in Chinese subjects. Following the first study, other investigators have examined the association of ACE gene polymorphism with OSAS using a larger sample of Chinese subjects [3]. The frequency of I allele and II genotype were significantly higher in the moderate to severe OSAS group than in the other groups. Further, the higher frequency of ACE gene I allele and II genotype was closely associated with the hypertensive patients with OSAS. They indicated that the inherited factors including ACE II genotype played an important role in the pathogenesis of hypertensive patients with OSAS.

However, it has been suggested that ACE DD genotype is associated with heart weight and hypertension in Japanese and Caucasian subjects [4, 5]. Further comparative studies concerning the association of ACE gene polymorphism with cardiovascular complications of OSAS between Asians and Caucasians are needed.

Because cardiovascular disorders are known to increase among patients with OSAS [6-8], the genetic susceptibility to hypertension and cardiac hypertrophy may exist in a population with OSAS. As the predictors of cardiovascular complications in patients with OSAS are not yet conclusively determined, another screening strategy is necessary to determine the high-risk group for cardiovascular disorders among OSAS patients. The risk of future cardiovascular diseases may be associated with the genetic susceptibility to cardiovascular disorders in OSAS patients. Thus, it is important to know whether the genetic background of OSAS patients with or without cardiovascular diseases exists. It has recently been reported that a significant portion of sleep-disordered breathing is associated with apolipoprotein (Apo)E epsilon 4 in the general population [9]. Because sleepdisordered breathing occurs in Alzheimer-disease patients and increases the risk for cardiovascular disease, complex interactions among sleep, brain pathology, and cardiovascular disease may occur in ApoE epsilon4 carriers. However, the other candidate gene polymorphisms associated with OSAS and OSAS related cardiovascular diseases are not extensively examined.

Because ethnic differences in the prevalence of obstructive sleep apnoea syndrome exist, further confirmation of the candidate gene polymorphisms and the association with obstructive sleep apnoea syndrome is needed, in both Caucasians and Asians using a large sample size [10].

\section{S. Teramoto*, H. Kume*, T. Matsuse ${ }^{\#}$, T. Ishii ${ }^{\#}$, Y. Inoue}

*International University of Health and Welfare, Sanno Hospital, Akasaka, Minato-ku, "Dept of Pulmonary Medicine, Yokohama City University Medical Centre, and "Dept of Psychiatry, Juntendo University School of Medicine, Tokyo, Japan.

\section{References}

1. Barcelo A, Elorza MA, Barbe F, Santos C, Mayoralas LR, Agusti AG. Angiotensin converting enzyme in patients with sleep apnoea syndrome: plasma activity and gene polymorphisms. Eur Respir J 2001; 17: 728732 .

2. Xiao Y, Huang X, Qiu C. Angiotension I converting enzyme gene polymorphism in Chinese patients with obstructive sleep apnea syndrome. Zhonghua Jie He Не Hu Xi Za Zhi 1998; 21: 489-491.

3. Zhang J, Zhao B, Gesongluobu SY, et al. Angiotensinconverting enzyme gene insertion/deletion (I/D) polymorphism in hypertensive patients with different degrees of obstructive sleep apnea. Hypertens Res 2000;23: 407-411.

4. Nakahara K, Matsushita S, Matsuoka $\mathrm{H}$, et al. 
Insertion/deletion polymorphism in the AngiotensinConverting enzyme gene affects heart weight. Circulation 2000; 101: 148-151.

5. Hamon M, Amant C, Bauters C, et al. Association of angiotensin converting enzyme and angiotensin II type I receptor genotypes with left ventricular function and mass in patients with angigraphically normal coronary arteries. Heart 1997; 77: 502-505.

6. Teramoto S, Kume H, Matsuse T, Fukuchi Y. The risk of future cardiovascular diseases in the patients with OSAS is dependently or independently associated with obstructive sleep apnoea. Eur Respir $J$ 2001; 17: 573-574.

7. Teramoto S, Ohga E, Ouchi Y. Obstructive sleep apnoea. Lancet 2000; 355: 1213-1214.

8. Barcelo A, Elorza MA, Barbe F, Vila M, Pons S, Agusti AG. Abnormal lipid peroxidation in patients with sleep apnoea. Eur Respir J 2001; 16: 644-647.

9. Kadotani H, Kadotani T, Young $\mathrm{T}$, et al. Association between apolipoprotein $\mathrm{E}$ epsilon4 and sleepdisordered breathing in adults. JAMA 2001; 285: 2888-2890.

10. Teramoto S, Ishii T. No association of tumor necrosis factor-alpha gene polymorphism and COPD in Caucasian smokers and Japanese smokers. Chest 2001;119: 315-316.

\section{From the authors:}

We thank S. Teramoto and colleagues for their interest in our paper, and we would like to respond to some of their comments. Although we explored angiotensin converting enzyme (ACE) gene polymorphisms in patients with obstructive sleep apnoea syndrome (OSAS), our paper focused on the ACE plasma activity in these patients. We think that its major contribution was to show increased ACE activity in their plasma, as compared to healthy controls. We stated in several paragraphs of our paper that the studies on prevalence of the different polymorphisms of the ACE gene have to be carried out in larger populations. Our data does not exclude a possible pathogenetic role of mutations of the ACE gene in OSAS patients, but we believe that genetic studies based on relatively small populations must be interpreted with caution. It is, therefore, difficult to accept, as suggested by $\mathrm{S}$. Teramoto and colleagues, that the frequency of the I allele and the II genotype are higher in Chinese patients with moderate to severe OSAS based on a population of only 34 individuals.

Another point to take into account in this discussion is the possibility that the association between ACE gene polymorphisms and OSAS may be different in Caucasian and Asiatic individuals; we agree with $\mathrm{S}$. Teramoto and colleagues that further confirmation is needed in both populations.

Finally, we agree that the risk of developing cardiovascular complications in patients with obstructive sleep apnoea syndrome may be associated with a genetic susceptibility. However, we are just beginning to uncover this field, and results must be evaluated with caution due to the potential bias in these studies. For example, two recent large studies have published apparently contradictory results about the relationship of apoE epsilon 4 to sleep breathing disorders $[1,2]$. Thus, any confirmation or exclusion of the possible connection between candidate gene polymorphisms and obstructive sleep apnoea syndrome remains unproven and requires large prospective studies.

\author{
A. Barcelò*, F. Barbé ${ }^{\#}$, A.G.N. Agustí ${ }^{\#}$ \\ *Servei de Pneumologia and ${ }^{\#}$ Anàlises Cliniques, Hospi- \\ tal Universitari Son Dureta, Palma de Mallorca, Spain.
}

\section{References}

1. Saarelainen S, Lehtimäki T, Kallonen E, Laasonen K, Poussa T, Nieminen MM. No relation between apolipoprotein E alleles and obstructive sleep apnea. Clin Genet 1998; 53: 147-148.

2. Kadotani H, Kadotani T, Young T, et al. Association between apolipoprotein $\mathrm{E}$ epsilon 4 and sleep disordered breathing in adults. JAMA 2001; 285: $2888-2890$ 